CASE XI.

First Admission (December 27th, 1914)-Digns of infection : -(1) Scar of sore on glans penis;'(2) slight general adenitis: (3) mucous patches on tongue and on right tonsil. No other visible sigu. Incubation period uncertain. Treatment: Eight injections of $0.3 \mathrm{gram}$ salvarsan and six weekly injections of 1 grain mercury cream ; completed February 23rd, 1915.

Second Admission (January 26th, 1916).-Signs of infection : (1) Two primary papular erosive syphilitic sores beneath prepuce; (2) shotty glands in groins. No other visible sign. Last exposure to infection five weeks before admission. Dark-ground examination, s. pallida present. Treatment: Eight injections of $0.3 \mathrm{gram}$ salvarsan with intervals, and seven weelily injections of mercury; completed March 21st, 1916.

CASE XIr.

First Admission (July 4 th, 1915).--Signs of infection: Noninflammatory syphilitic oedema of skin of penis, hard nodule felt beneath phimosed prepuce. No other visible sign. Incubation period ten days. Dark-ground examination, s. pallida present. Treatment: Eight injections of 0.3 gram salvarsan and six weekly injections of 1 grain mercury cream; completed August 17th, 1915.

Second Admission (June 15th, 1916).--Signs of infection: (1) Primary papular erosive svphilitic sore on inner surface of prepuce; (2) marked adenitis in both groins. Scar of old sore present. Incubation period three weeks. Wassermann test incomplete, Stern positive. Treatment: Eight injections of 0.3 gram salvarsan with intervals, and five weekly injections of 1 grain mercury cream; completed August 7 th, 191.6.

CASE XIII.

First Admission (November 5th, 1915).--Signs of infection: (1) Typical Hunterian chancre at fraenum; (2) adenitis in groins and neck. No other visible sign. Incubation period one month. Treatment: Eight injections 0.3 gram salvarsan with intervals, and seven weekly injections of 1 grain mercury cream ; completed February 8th, 1916.

Second Admission (July 8th, 1916).--Signs of infection: (1) Typical Hunterian chancre on right inner surface of prepuce (2) adenitis in groins. No other visible sign ; scar of previous sore at fraenum. Last exposure to infection about five weeks before admission. Wassermann and Stern tests negative. Treatment: Same as on first admission; completed August 24th, 1916 .

Case Xiv.

First Admission (September 22nd, 1915).-Signs of infection : Two primary papular erosive syphilitic sores, one on inner surface of prepuce, one close to fraenum. No other visible sign. Incubation period not reliable. Dark-ground examination, $s$. pallida present. Treatment: Eight injections of 0.3 gram salvarsan and five weekly injections of 1 grain mercury cream ; completed December 6th, 1915.

Second Admission (July 16th, 1916). - Signs of infection : (1) Typical Hunterian chancre in coronal sulcus just to right of dorsal middle line; (2) general adenitis and a right bubo; (3) papular squamous rash over body and limbs; (4) mucous patch on inner side of upper lip. Scar of old sores present. of $0.3 \mathrm{gram}$ salvarsen with intervals, and six wight injection of 1 grain mercury cream; completed August 30th, 1916.

CASE Xv.

First Admission (November 6th, 1915).-Signs of infection : (1) Primary papular erosive syphilitic sore at fraenum ; (2) indurated glands in groins. No other visible sign. Last exposure to infection five weeks before admission. Dark-ground examination, S. pallida present. Treatment: Eight injeetions of 0.3 gram salvarsan and five weekly injections of 1 grain mercury 0.3 gram salvarsan and five weekly injecti

Second Admission (December 19th, 1916).-Signs of infection: (1) Primary Hunterian chancre in coronal sulcus; second primary granulating syphilitic sore on under surface of prepuce; (2) glands in both groins enlarged and hard. No other visible sign. Last exposure to infection about six weeks before admission. Wassermann test positive. Treatment: Three injections of $0.3 \mathrm{gram}$ salvarsan, one of $0.4 \mathrm{gram}$ three of 0.5 gram with intervals, and five weekly injections of 1 grain mercury cream. Treatment completed February 15th, 1917, when the Wassermann reaction was negative.

\section{CASE Xvi.}

First Admission (January 9th, 1915).--Signs of infection : (1) Typical Hunterian chancre on inner surface of prepuce; (2) roseolar syphilide on chest. No other visible sign. Incubation' period one month. Treatment: Four injections of 0.3 gram salvarsan and eight weekly injections of 1 grain mercury cream ; completed March 2nd, 1915. On March 7th, 1916, the patient was readmitted with ulceration of both tonsils no other visible signs. He was given a full course of eight injections of 0.3 gram salvarsan with intervals, and tive weekly injections of 1 grain mercury cream. He was discharged to duty on May 3rd, 1916.

Second Admission (October 2nd, 1916).-Signs of infection : (1) Early primary papular erosive syphilitic sore in left of coronal sulcus; (2) glands in groins painlessly enlarged and hard and shot-like. No other visible sign. Incubation period two weeks. Treatment: Three injections of $0.3 \mathrm{gram}$, one of $0.4 \mathrm{gram}$, and three of 0.5 gram salvarsan with intervals, and six weekly injections of I grain mercury cream; completed December 9th, 1916. The Wassermann reaction was incomplete both before and after treatment.

Case Xvir

First Admission (December 4th, 1915). Signs of infection: (1) Balanitis, a hard nodule to be felt under foreskin about level o fraenum; (2) glands in groin shot-like and enlarged. No other visible sign. Last exposure to infection about six weeks before admission. Treatment: Eight injections of 0.3 gram salvarsan with intervals, and seven weekly injections of 1 grain mercury cream ; completed January 25th, 1916.

Second Admission (April 12th, 1917).- Signs of infection: (1) A typical primary papular erosive syphilitic sore occupied fraenal site ; (2) painless shotty adenitis in left groin. No other visible sign. Incubation period one month. Dark-ground examination, $S$. pallida present. Treatment: Seven weekly deep subcutaneous injections of 0.6 gram salvarsan and five weekly injections of 1 grain mercury cream; completed May 28th, 1917. The Wassermann reaction was negative both before and after treatment.

CASE Xvirr.

First Admission (May 22nd, 1915).--Signs of infection: (1) Small papular erosive syphilitic sore on under surface of fore skin ; (2) glands in both groins enlarged; (3) macular rash on body. No other visible sign. Incubation period one month. Treatment: Eight injections of 0.3 gram salvarsan and six weekly injections of 1 grain mercury cream; completed July 13th, 1915.

Second Admission (March 2nd, 1917).- Signs of infection : (1) Phimosis, unable to retract foreskin; a clean indurated granulating syphititic sore on anterior surface of skin of penis; (2) glands in groins painlessly enlarged and shot-like. No othe visible sign. Incubation period eighteen days. Treatment Six weekly injections of 0.6 gram salvarsan, deep subcutaneous; five weekly injections of 1 grain mercury cream. Treatment completed May 1st; 1917. The Wassermann reaction was negative both before and after treatment.

The cases in this series are not supported by the bacteriological findings and serum tests, and so do not comply with the conditions laid down for Series I, still the likelihood of the correctness of the diagnosis is enhanced by the fact that again, as in the cases given in Series I, the two distinct attacks of syphilis have been seen in all the cases here recorded by the same observer. The cases were seen, the notes checked, and the treatment of each patient carried out by me at the time of his first and second attack.

The above 28 cases of reinfection occurred in a series of 10,500 cases of syphilis treated in No. - General Hospital during the past two years.

For the Wassermann tests and dark-ground examinations I am indebted to Captains A. Dawson and A. T. MacWhirter, R.A.M.C., and I wish to thank Sergeant G. T Platford, R.A.M.C., for the trouble he has taken in summarizing the notes on the cases given in Series I and II.

THE MEDICINAL TREATMENT OF GONORRHOEA.

BX

Major H. C. DONALD, M.B., C.M., F.R.C.S.Edin, R.A.M.C.(T.F.)

AND

Captain A. M. Davidson, M.B., Ch.B.Edin., M.D.MANITOBA, R.A.M.C.

A QUESTION which was frequently asked by many medical officers who visited a certain venereal hospital for the purpose of inquiring into the routine methods of treat. ment was, "Is irrigation into the bladder any better than flushing of the urethra from within by the use of medicines?" With a view to deciding this question permission was granted to treat cases with medicine alone.

Routine treatment as carried out generally in the above mentioned hospital in ordinary cases meant three irrigations a day into the bladder, prostatic massage, and instrumental treatment where indicated.

Method of Treatment.

The intention at first was to have a control series of one hundred patients on routine irrigation treatment and another series of one hundred patients on medicinal treat. ment. When, however, volunteers were called for every patient volunteered for the medicinal treatment, and it was then decided, in order to have the full confidence of the patients, to give every patient medicinal treatment. Accordingly the whole division, consisting of about two 
hundred patients, commenced the treatment, which consisted of diuretics in the early stages, chiefly Fourniër's formulae and the usual potassium citrate, hyoscyamus, and buchu mixture, and in the later stages mixtures containing the oleo-resins (oil of copaiba, sandal wood oil, and oil of cinnamon): Fournier's formulae were as follows :

$$
\begin{aligned}
& \text { 1. Nitrate de potasse ... } \quad \ldots . . .60 \text { gram } \\
& \text { Douce-amere (woody nightshade, } \\
& \begin{array}{lllll}
\text { Solamum dulcamara) } \quad \ldots & \ldots & 10.00 \text { grams }
\end{array} \\
& \text { Chien dent (Ayropyrum repiens) } \quad \ldots .10 .00 \text {," } \\
& \text { Infused in a litre of water. }
\end{aligned}
$$

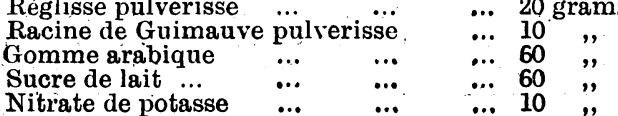

Make in doses of 10 grams per litre of water, to be taken in twenty-four hours.

The medicine was taken six times a day at the following hours: 6 a.m., 8 a.m., 11 a.m., 2 p.m., 5 p.m., and 8 p.m.

The patients paraded under their respective sergeants, marquee by marquee, at the above hours, and as they filed into the treatment hut they received their medicine in their basins, and, after passing belind the irrigation trough, they placed their basins on the shelf. The drinking of the medicine was carried out under the strict supervision of the wardmaster, so that no one missed taking hia dose. After taking the medicine, the patients washed the glans penis and prepuce by means of swabs soaked in a solution of potassium permanganate contained in the irrigation buckets, and afterwards they washed their hands.

They received no other treatment except when urethral infiltrations were present, in which cases dilatation was carried out. No vaccine or prostatic massage was givèn.

The patients were weighed every six days in order to ascertain if the imbibing of large quantities of fluid affected their fitness for duty. It; liowever, made no appreciable difference.

\section{Clinical Observations.}

Compared with irrigation treatment, it was found that: Discharge.-The urethra remained purulent for a much longer period and never became " bone dry."

Condition.-Oedema persisted for a longer time.

Pain.-Complaints of pain persisted for weeks. Severe pain was complained of in many more cases than if the patients had been on irrigation treatment. "This was very. striking.

Frequency.-Owing to the large amount of fluid imbibed frequency of micturition was naturally greater (ten to twenty times a day on the average).

Bovels.-These were naturally looser than usual.

\section{Progress and Complications.}

More cases became subacute and chronic, and seemed to have a chronic oedema, which does not occur under irrigation treatment. There were more palpable periurethral infiltrations in the form of follicles and periurethritis. There was less tendency for the discharge to cease and the urethra to become dry than under irrigation treat ment. The routine urine test did not show the same progress. In spite of the large amount of fluid taken, shreass were easily observed in the urine, which would suggest that there was more posterior urethritis. The treatment did not prevent the development of the same percentage of epididymitis (acute and subacute) as occurs under irrigation treatment. The same was true of arthritis.

After carrying out this treatment for two months we were obliged to resort to the old methods of treatment, and it was noticeable that many resistant cases cleared up rapidly. With dilatation the subacute inflammation of the urethra quickly subsided. Pus could still be massaged out of the prostate after weelss of medicinal treatment.

\section{Statistics.}

At the time of returning to irrigation treatment it was found that there were more chronic cases in the division than ever before. Only 78 men had been discharged to duty on medicine alone in the period of two months, as against four times as many discharged in a similar period under irrigation treatment. Of these 78 men, 65 per cent. were cases of relapse either after treatment in this hos. pital or other hospitals. Only 24 per cent. were primary infections.

As far as can be at present ascertained, the relapse rate for this series of men discharged aftei medicinal treatment only is already higher than that of any other series.

The effect of -medicine in prolonging the stay in hospital is clearly shown by a comparison between two series of similar composition-one series treated on the routine irrigation system, and the other by both irrigation and medicine (but not both together).

It was found that in the latter series the number of days in hospital for all the cases was increased by 14 ; the treat. ment of primary infections was lengthened by 18 days; the treatment of subsequent infections by 20 days; and the treatment of relapses from this hospital. or other hospitals by 7 days.

\section{Conclusion.}

To sum up, medicinal treatment from a clinical and statistical point of view undoubtedly does not help but retards the cure of gonorrhoea, and tends to produce chronicity.

\section{OPERATIONS ON \\ THE NASAL SINUSES CARRIED OUT THROUGH A TEMPORARY OPENING IN THE SEPTUM (TRANS-SEPTAL).}

By NORMAN PATTERSON, M.B., B.CH., F.R.C.S. ASSISTANT SURGEON, LONDON HOSPITAL; SURGEON, THROAT HOSPITAI. GOLDEN SQUARE, ETC.

There are two routes by which the ethmoidal cells may be approached. By far the most usual is through the nose; only in rare instances is the disease attacked from the ontside through a skin incision. The external operation may be adopted in cases where there is an ethmoidal mucocele, suppurating or otherwise, causing a swelling in the neighbourhood of the inner canthus; in dealing with a diseased ethmoidal labyrinth and frontal sipus at one and the same time; in removal of tumours, innocent or malignant (for example, exostosis, sarcoma); and, in rare mstances, where a very thorough exposure of the ethmoidal cells is called for. The external operation has the advantáge that anatomical landmarks can be more clearly defined, and the diseased cells can be dealt with under direct vision. Its main disadvantage is that a scar is left on the face, and that normal non-infected tissues are of necessity opened up. The advantage of the nasal route of approach is that there is no resulting scar, and that the infected area is not reached through liealthy tissues. Its main disadvantage is that it is impossible to get a really good view of the field of operation. Much depends on guess work and the "feel" of the tissues under" the instrument.

I liave endeavoured to improve on the route of approach to the ethmoidal cells, and, incidentally, to the spbenoidal sinus, by adopting the plan here described.

In viewing the region of the middle turbinate and ethmoidal labyrinth from the corresponding nostril, one is met with the difficulty that it is impossible to obtain a direct view of the field of operation.

The anterior end and a portion of the lower border of the middle turbinate is brought well into the field, but, even after this structure has been removed, only a very unsatisfactory view of the ethmoidal region is obtained. It occurred to me that the structures in this, neighbourhood might be brought, so to speak, "under the eye of the surgeon" by approaching them through the opposite nostril. (No one can have failed to remark on the excellent display of the opposite side of the nose revealed through a large septal perforation.) The view becomes much more direct, and, in the case of the anterior ethmoidal region at any rate, a more or less surface view becomes possible (see Fig. 1). To obtain this view it is necessary, of course, to make an incision through the septum, and it is of importance that the septal aperture be only a temporary one.

I shall now describe the various methods I liave tried in making this temporary passage through the septum. 